\title{
La pollution des Baies de Morlaix et de Lannion par les hydrocarbures de 1" "Amoco Cadiz": Répartition sur les fonds et évolution
}

\author{
A. Beslier ${ }^{1}$, J. L. Birrien ${ }^{2}$, L. Cabioch $^{2}$, C. Larsonneur ${ }^{1}$ \\ \& L. Le Borgne ${ }^{2}$ \\ ${ }^{1}$ Laboratoire de Géologie Marine, Université de Caen; \\ F-14032 Caen Cedex, France, \\ et \\ ${ }^{2}$ Station Biologique; F-29211 Roscoff, France
}

\begin{abstract}
Distribution of hydrocarbons from the "Amoco Cadiz" oil spill in sublittoral sediments, north of Brittany. The quantitative distribution of hydrocarbons in the sublittoral sediments, north of Brittany (France), was followed and recorded for the first year after the spill (March, 1978). Following preliminary observations in spring of 1978, 250 stations were sampled in August from the Bay of Lannion to Portsall at water depths down to $70 \mathrm{~m}$. Subsequently, 40 stations were studied at three-month intervals. During the summer of 1978, the widest polluted sublittoral areas were localised in the Bays of Morlaix and Lannion down to depths of about $30 \mathrm{~m}$ and even deeper in some places. In these two bays, the distribution of hydrocarbons showed: (1) deposition near the coasts hit by the slicks, mostly after adsorption by fine sedimentary particles; (2) redistribution of particulate and adsorbed oil towards low hydrodynamic energy areas favourable to deposition of fine particles. In autumn and winter, the coarse sediments became progressively less polluted. Oil, often in significant quantities, remained or was reconcentrated in the fine sediments of the inner bay parts. Consequently, the hydrodynamic structure ot the area (waves, tidal currents) has played an important role in the deposition and redistribution of the hydrocarbons on sublittoral bottoms.
\end{abstract}

\section{INTRODUCTION}

Dans la nuit du 16 au 17 mars 1978, le super-pétrolier libérien "Amoco Cadiz" s'échouait sur la côte nord de Bretagne, à $2 \mathrm{~km}$ au large de Portsall. En 2 semaines, sa cargaison de 223000 tonnes de brut se déversait à la mer engendrant la pollution catastrophique d'une bande côtière d'environ $250 \mathrm{~km}$.

Poussées par le vent, accrochées par les rochers, soumises au balancement des marées, localement traitées par des absorbants ou des dispersants, les nappes d'hydrocarbures ont progressivement atteint les plages, l'intérieur des estuaires et des abers et pénétré en profondeur dans l'etage infralittoral, voire même circalittoral. Les baies de Morlaix et de Lannion situées entre 60 et $100 \mathrm{~km}$ du point d'échouage, à l'Est, ont été particulièrement touchées. Nous y avons entrepris un programme de recherches consacré principalement au domaine subtidal et complétant ainsi les travaux accomplis sur la côte par d'autres équipes. 


\section{OBJECTIF ET METHODES}

Dès le début avril 1978, les premières campagnes de surveillance signalent la présence d'hydrocarbures dans les sédiments subtidaux des baies de Morlaix et de Lannion (Cabioch et al. 1978; Marchand \& Caprais, 1979). En mai-juin, plusieurs zones se révèlent contaminées, une collaboration s'organise alors pour cartographier précisément la pollution, examiner ses relations avec la répartition et la nature des sédiments, déterminer son impact sur les peuplements benthiques subtidaux. Cette collaboration regroupe d'une part la station biologique de Roscoff et le Laboratoire de Géologie marine de l'Université de Caen, d'autre part le Centre Océanographique de Bretagne (Brest) et l'Institut de Recherches de l'Université de Columbia (Caroline du Sud-USA).

Une importante campagne de prélèvements et d'observations se déroule en juillet août 1978. 226 échantillons sont recueillis à la benne Hamon, le tout complété de photographies et d'études directes par télévision sous-marine. Cette campagne constitue la base de référence pour les observations de suivi ultérieures accomplies pour 40 stations en novembre 1978, février et mai 1979. Une couverture complète de la région vient d'être refaite en juillet-août 1979 , soit un an après notre point 0 , les résultats n'en sont pas encore disponibles. Pour compléter nos travaux, quelques prélèvements de comparaison ont également été faits et suivis dans la zone intertidale.

L'échantillonnage réalisé se situe presqu'entièrement au-dessus de l'isobathe 50 , c'est à dire sur la ceinture subcôtière très accidentée prolongeant la morphologie complexe du littoral et dominant les étendues plates et monotones du large. Les fonds très variés dessinent une mosaique irrégulière où la benne Hamon par sa grande surface de prélèvement $\left(0,28 \mathrm{~m}^{2}\right)$ et sa bonne pénétration s'est révélée bien adaptée et efficace. Dans ces zones tourmentées, une attention particulière a été apportée à la rigueur du positionnement.

\section{L'analyse des hydrocarbures dans les sédiments}

Le sédiment prélevé est stocké dans un récipient en verre préalablement nettoyé à l'eau distillée puis séché et lavé au tétrachlorure de carbone. Les échantillons sont congelés et conservé à $-20^{\circ} \mathrm{C}$. Les dosages réalisés ont été effectués selon le protocole suivant.

Le sédiment est séché à $60^{\circ} \mathrm{C}$ pendant 48 heures dans une étuve ventilée. A $5 \mathrm{~g}$ de matériel sec on ajoute $20 \mathrm{ml}$ de tétrachlorure de carbone et on soumet aux ultrasons (120 W) pendant 10 min dans un bain d'eau glacée afin d'éviter tout réchauffement. Après décantation du surnageant, on ajoute du sulfate de sodium afin de parfaire la déshydratation puis on filtre sur une bourre de laine de verre. On réajuste le volume du filtrat à $20 \mathrm{ml}$ avec du tétrachlorure de carbone et on enregistre le spectre sur un spectrophotomètre infra-rouge (Unicam SP 1100) entre $2500-3500 \mathrm{~cm}^{-1}$ correspondant à la zone d'absorption des hydrocarbures totaux (alcanes, alcènes, alcynes et aromatiques extractibles par le tétrachlorure de carbone) d'après Beynon et al. (1968) et la norme API $733.58 \mathrm{du}$ CNEXO (Causse \& Mestres, 1975). La courbe étalon à été établie à partir d'une solution d'Iranian light étêtée à $150^{\circ} \mathrm{C}$, dissoute dans du tétrachlorure de carbone pour spectroscopie. Pour le dosage des hydrocarbures aromatiques nous avons adopté une méthode spectrofluorimétrique (Hiltabrand, 1978) utilisant le n-Hexane comme solvant avec le même protocole d'extraction que pour l'infra-rouge. La courbe étalon a été faite 
dans le n-Hexane, les lectures ont été effectuées au maximum d'excitation à $310 \mathrm{~nm}$; la fluorescence est lue à $370 \mathrm{~nm}$. Les extractions sont poursuivies avec le solvant approprié jusqu'au seuil de sensibilité de l'appareil : $10 \mathrm{ppm}$ pour l'infra-rouge et $1 \mathrm{ppm}$ pour la spectrofluorimétrie. Le nombre des extractions est conditionné par le pouvoir de rétention de chaque type de sédiment en relation avec sa composition physico-chimique. Des essais d'extraction ont été faits sur des échantillons de sédiment non pollué auxquels avait été jointe une quantité connue d'hydrocarbures. Le taux de récupération dans les sables est de l'ordre de $90 \%$; il est plus faible dans les sédiments vaseux. Néanmoins, dans tous les cas la reproductibilité des mesures est bonne.

\section{Cartographie des sédiments}

La méthode utilisée est celle mise au point pour la réalisation de la carte des sédiments superficiels de la Manche au 1/500 000 (Larsonneur et al., 1978; Larsonneur, 1977); elle implique l'analyse granulométrique et calcimétrique des sédiments recueillis. Les coupures 30,50 et $70 \%$ de calcaire permettent de distinguer respectivement les dépôts lithoclastiques, litho-bioclastiques, bio-lithoclastiques et bioclastiques tandis que les subdivisions classiques en cailloutis, graviers, sables grossiers, sables fins, sablons et vases sont retenues avec tous leurs intermédiaires. Les surfaces rocheuses ont été délimitées à partir des cartes du Service Hydrographique et Océanographique de la Marine et le document synthétique obtenu a été élaboré en tenant compte des travaux antérieurs principalement consacrés à la baie de Morlaix (Boillot, 1964; Anonymous, 1968; Cabioch, 1968).

La carte (Fig. 1) montre la présence de fonds rocheux associés à des sédiments grossiers lithoclastiques ou litho-bioclastiques dans les zones externes, vers 30 à $50 \mathrm{~m}$ de profondeur. Prennent également place dans ces régions de grands corps sableux essentiellement formés de débris coquilliers (sables fins, moyens ou grossiers, souvent graveleux bioclastiques ou bio-lithoclastiques). Les sédiments s'affinent à l'approche du rivage, notamment dans les baies où se rencontrent sables fins, sablons accompagnés localement de dépôts vaseux. Formé d'une fine mouture organogène, le matériel est souvent très carbonaté; de 60 à $80 \%$ en général. Ce gradient granulométrique résulte de l'amortissement progressif des courants de marée du large vers les échancrures du littoral. Près du rivage, l'action des houles devient prépondérante; platiers rocheux, plages de sable fin, cordons de galets constituent la mosaïque des fonds côtiers. Le matériel vaseux se répartit largement dans les estuaires, il est également incorporé à certains sédiments phycogènes des baies de Morlaix et de Lannion.

\section{RESULTATS}

La pollution des Baies de Lannion et de Morlaix par les hydrocarbures: L'état en août 1978

\section{Observations initiales}

Libérées des cuves de l' "Amoco Cadiz" et soumises à un régime de vents violents de secteur ouest, les nappes de mazout se sont rapidement étalées vers l'Est atteignant l'ensemble de la région les 20 et 21 mars. Sur une frange côtière de plus de $100 \mathrm{~km}$ dans 


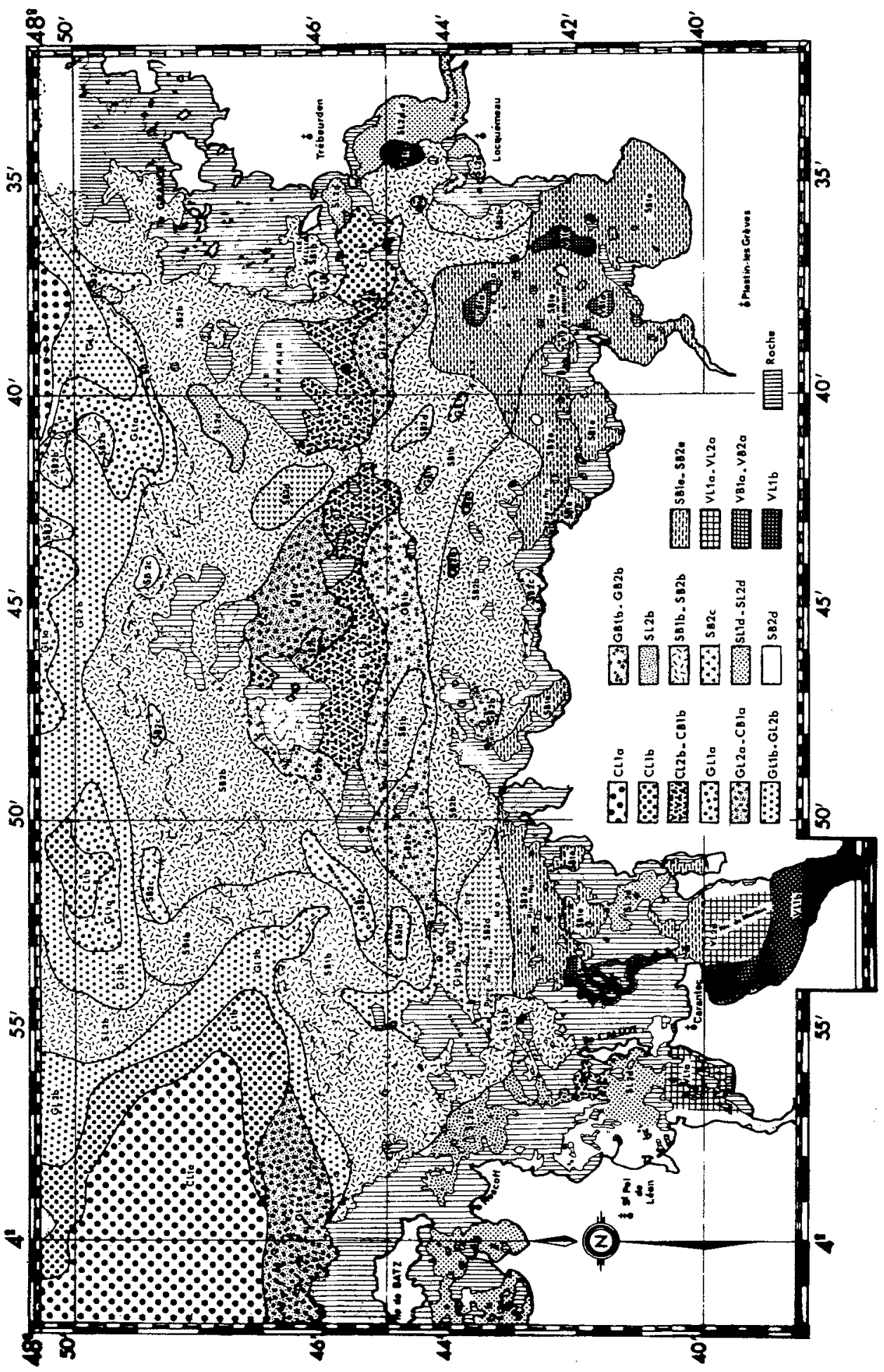

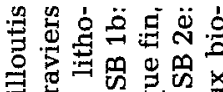

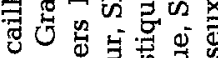
तิ

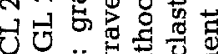

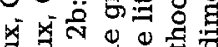

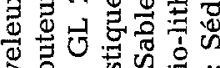

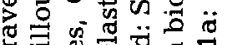
G 3 궁

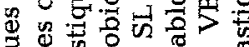

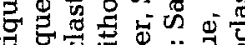

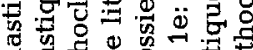

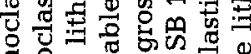

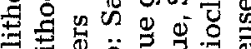

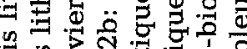

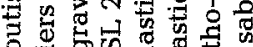

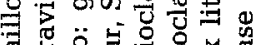

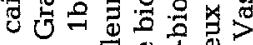

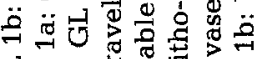
Uण

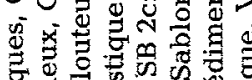

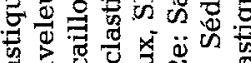
ช

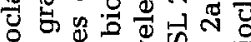

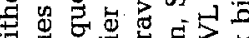
ज部

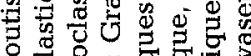
要

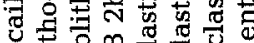

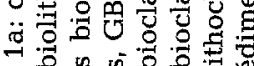

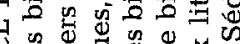
U.

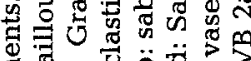

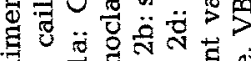

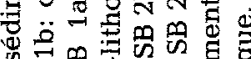
政

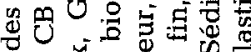
당

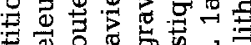
\% 过客记 的

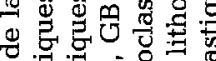
㻤 ช च चु - 웅웅

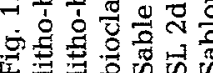


La pollution des Baies de Morlaix et de Lannion

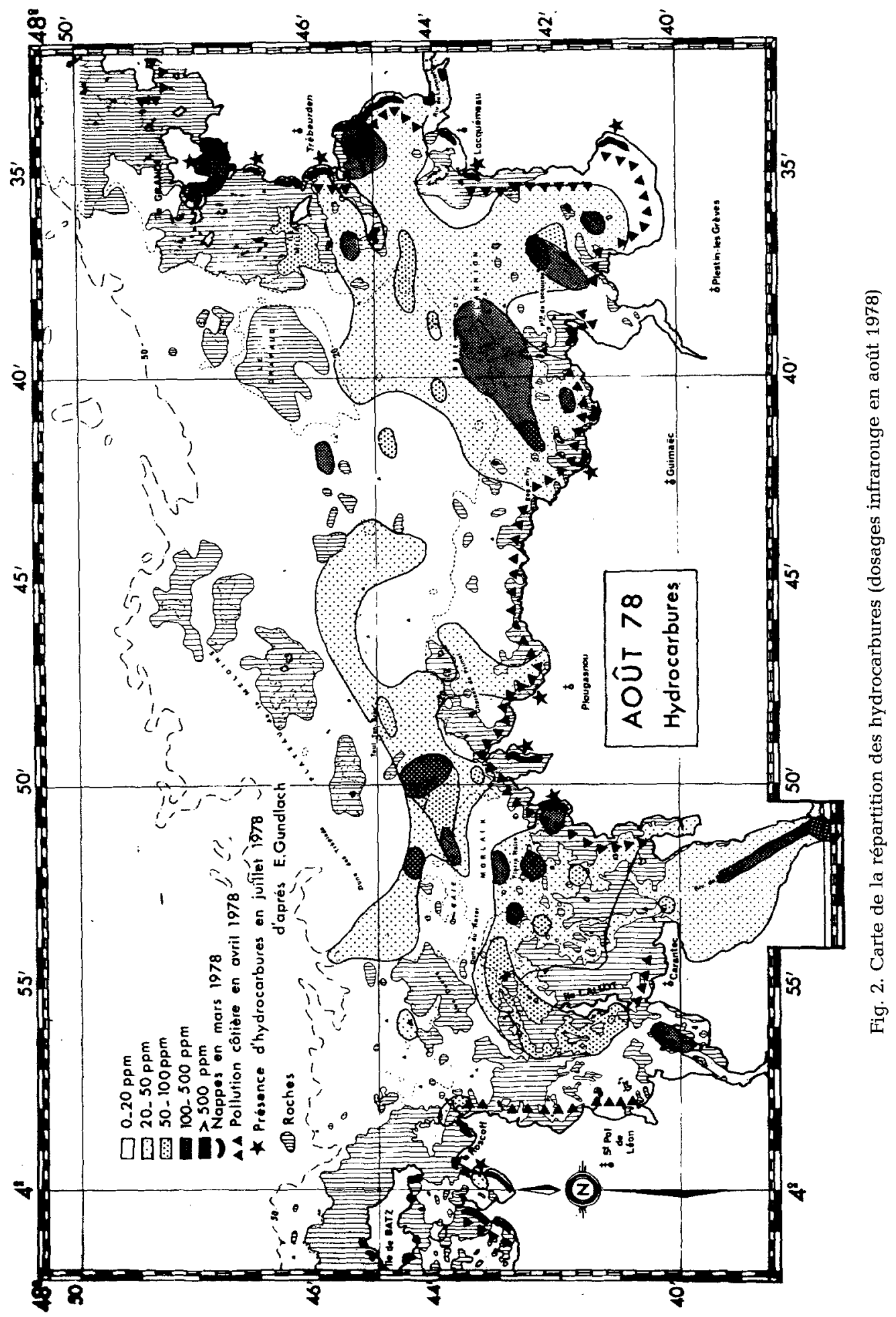


une zone très accidentée, les hydrocarbures se sont trouvés accumulés et placés durant les premières semaines dans des conditions de forte agitation. Il est connu qu'une dispersion du pétrole dans l'eau est alors susceptible de se produire, elle peut être suivie de son absorption sur des particules sédimentaires en suspension (MacAuliffe, 1977, Huang \& Elliott, 1977). Il en résulte, d'une part une stabilisation de l'émulsion d'huile dans l'eau, d'autre part le dépôt de ces particules oléo-sédimentaires sur les fonds quand l'agitation décroît. L'action éventuelle des dispersants et absorbants utilisés pour traiter les nappes en mer est également à envisager dans ces phénomènes. Dans ces conditions, le mazout a non seulement pollué la zone intertidale mais il a pénétré dans les sédiments subtidaux. Cette particularité très importante de la marée noire de l'Amoco Cadiz fut observée pour la première fois le 3 avril dans les sables très fins de la Pierre Noire au NE de la baie de Morlaix par $18 \mathrm{~m}$ de profondeur (Cabioch et al., 1978).

Elle fut constatée peu après, courant avril, en divers points des baies de Morlaix et de Lannion et même jusqu'à $70 \mathrm{~m}$ de profondeur dans les sables coquilliers du Trezen Vraz (Marchand \& Caprais, 1979). Les chenaux subtidaux des rivières de Morlaix et de la Penzé étaient également atteints alors qu'il n'y était pas parvenu de grande nappe par la surface. Un processus de transfert au niveau du fond ou dans la colonne d'eau avait donc eu lieu, suivi d'une sédimentation préférentielle dans les zones les plus calmes.

En mai 1978, l'utilisation de la télévision sous-marine confirmait les précédentes observations. Des placages sombres, irréguliers, de matériel oléosédimentaire se distinguaient nettement à la surface des sables fins. Par endroits, de petits amas bruns de "mousse au chocolat" (émulsion d'eau dans l'huile) apparaissaient; ailleurs les sables présentaient leur aspect habituel en surface, malgré une contamination en profondeur déterminée après prélèvement.

L'importante campagne de l'été 78 permit de délimiter les zones atteintes par les hydrocarbures et d'apprécier le degré de pollution.

\section{La répartition de la pollution en juillet-août 1978}

Les nombreux dosages d'hydrocarbures ont permis d'établir la carte de la Figure 2 qui donne la répartition des teneurs obtenues en spectrophotométrie infra-rouge. La plupart des échantillons ont également été dosés en fluorimétrie, les résultats sont voisins. Il apparaît que la pollution affecte principalement le fond de la baie de Morlaix et les abords de la Pierre Noire, la baie de Lannion et une large bande située entre les Chaises de Primel et le Plateau de la Méloine. Elle est également abondante près de l'Ile Grande, de l'Ile Milliau et dans la région de Roscoff près des zones d'échouage des nappes.

La comparaison avec la carte de répartition des sédiments montre que le plus souvent les hydrocarbures sont associés aux sédiments fins (dépôts vaseux et sablons). Les suspensions de particules oléo-sédimentaires semblent avoir une dynamique voisine des suspensions minérales de telle sorte qu'elles se concentrent dans les zones de faible énergie. Ainsi sont elles très abondantes dans les estuaires (Penzé, rivières de Morlaix et de Lannion) où les teneurs dépassent localement 1000 ppm. Dans la rivière de Morlaix, cette importante pénétration des hydrocarbures a entraîné la pollution des parcs à huîtres implantés sur les grèves sablo-vaseuses de Carantec. Plus à l'Ouest, des accumulations comparables, voire plus élevées, se sont produites dans les profonds abers de la région: Aber Wrach et Aber Benoît (Allen et al., 1978; Marchand \& Caprais, 
1979). Les hydrocarbures sont également abondants entre 5 et $20 \mathrm{~m}$ de profondeur, dans des zones de faible turbulence où se déposent sablons et sablons vaseux (Pierre Noire, baie de Lannion). Ces zones d'énergie minimum s'intercalent entre la frange côtière soumise à l'action croissante des houles au fur et à mesure que la tranche d'eau diminue et le domaine du large où l'influence des courants de marée devient prépondérante et s'accentue avec la profondeur. Dans ces conditions, dans l'axe des petites anses de la baie de Lannion par exemple trouve-t-on successivement du large vers la côte: roches et cailloutis, graviers, sables graveleux, sablons localement vaseux puis, sables fins sur les plages accompagnés de platiers rocheux (Fig. 1). Les zones d'énergie minimum situées à faible profondeur sont l'une des caractéristiques de l'hydrodynamisme en Manche où les courants de marée sont particulièrement violents (Larsonneur, 1972).

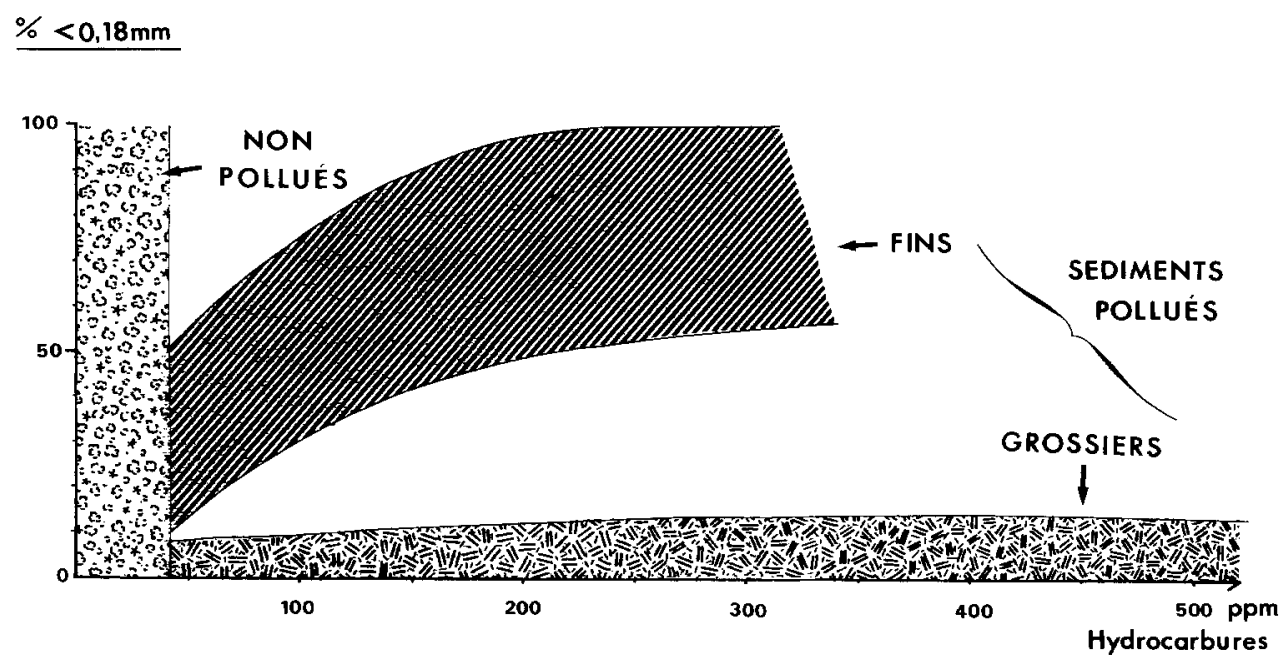

Fig. 3. Relation entre les teneurs en hydrocarbures et les teneurs en particules fines inférieures à $0,18 \mathrm{~mm}$ (en août 1978)

L'étude du rapport teneur en hydrocarbures - teneur en particules fines inférieures à $0,18 \mathrm{~mm}$ (sablons, silts et argiles) montre qu'une relation existe le plus souvent entre ces deux paramètres; les dépôts les plus fins étant les plus sensibles à la contamination (Fig. 3). Plus précisément, on constate graphiquement que les sédiments contenant plus de $50 \%$ de particules inférieures à $0,18 \mathrm{~mm}$ sont susceptibles d'être fortement pollués; les teneurs peuvent y devenir très élevées, jusqu'à plusieurs milliers de ppm. L'ensemble de ces résultats traduit un triage du matériel transporté par les agents hydrodynamiques locaux.

Cependant, les sédiments fins ne sont pas les seuls à être contaminés; certains fonds sableux ou plus grossiers, voire rocheux, sont également atteints: abords de l'île Grande et de l'île Milliau à l'Est, région sud du plateau de la Méloine, platier de Roscoff notamment. Il s'agit là de zones où les épaisses nappes de mazout, ballotées par la mer et placées ainsi dans des conditions favorables à leur émulsification dans l'eau se sont délestées au cours des semaines qui ont suivi la catastrophe. De la sorte, selon la 
répartition des apports par la marée noire, diverses catégories de sédiments subtidaux se sont trouvés contaminés par les hydrocarbures.

Enfin, ceux des dépôts de maerl qui constituent un piège biologique pour les particules fines le sont également pour les hydrocarbures qu'ils retiennent souvent en abondance: gisements de la partie NE de la baie de Lannion au large de Trébeurden et du milieu de la baie de Morlaix au voisinage de l'île Callot.

La Figure 2 donne en outre la répartition de la pollution intertidale en mars et en avril 1978 jointe à quelques observations de juillet 1978 d'après les études de d'Ozouville et al. (1978), Gundlach \& Hayes (1978, 1979), Hayes \& Gundlach (1979) et Berné et al. (1978). On constate que la pollution en domaine subtidal n'est pas directement calquée sur la répartition des nappes échouées à la côte. En milieu intertidal, la contamination résulte principalement d'apports par la surface étroitement liés aux dérives créées par le vent. En milieu subtidal, l'hydrodynamique locale joue un rôle important. En l'occurence, les courants de marée exercent une influence déterminante. $\mathrm{Au}$ large, leur vitesse dépasse presque partout 2 noeuds en surface, elle s'amortit progressivement vers l'intérieur des baies. Il s'agit de courants alternatifs dont les résultantes varient d'un point à un autre et ne sont pas encore complètement connues. En baie de Morlaix, les travaux d'Auffret \& Douvillé (1974) et de Douvillé (1975) montrent que les eaux de fond ont tendance à entrer par l'Est et à ressortir par le Nord-Ouest après avoir parcouru le fond de la baie. Plus au Nord, les dérives résiduelles qui longent la Bretagne portent à l'Est, dans le sens du courant général qui conduit les eaux océaniques vers la Mer du Nord. Cette tendance compensée par un mouvement contraire dans la partie nord de la Manche a été mise en évidence par flotteurs dérivants de fond (Jones, 1974), elle ressort également pour la zone étudiée des suivis de flotteurs effectués par Cabioch \& Douvillé (1979).

La campagne de carottages réalisée en août 78 en des stations fortement contaminées a montré que la percolation du pétrole en profondeur était faible, quelques centimètres en général (Berné, D'Ozouville \& Marchand: communication personnelle). Ce résultat rejoint ceux obtenus par Boucher (1979) dans les sables fins de la Pierre Noire et par Marchand \& Caprais (1979) sur les plages de la région de Portsall.

\section{L'évolution de la pollution jusqu' au printemps 1979}

Le suivi réalisé en novembre 1978, février et mai 1979 pour 40 stations remarquables des baies de Morlaix et de Lannion permet de dégager les principaux aspects de l'évolution de la pollution durant la première année (Fig. $4 \mathrm{a}, \mathrm{b}, \mathrm{c}$, et d).

En novembre 1978 (Fig. 4b), on constate une importante décontamination des zones de forte énergie. La pollution a disparu aux abords de Roscoff et dans presque toute la zone comprise entre le plateau de la Méloine et les Chaises de Primel. Dans les zones moins turbulentes de la baie de Lannion et de la Pierre Noire, la dépollution est également très sensible dans l'ensemble. Par contre, les teneurs en hydrocarbures restent très élevées localement, elles sont même plus élevées qu'en août sur les grèves sablo-vaseuses de Carantec en domaine estuarien et dans le maerl envasé de la région de Trébeurden-Locquémeau. Cette situation automnale fait suite à une longue période de temps assez calme avec agitation faible à modérée de la mer. Les observations faites au sémaphore de l'île de Batz montrent que de la fin avril au 15 novembre 78 l'état de la 
La pollution des Baies de Morlaix et de Lannion

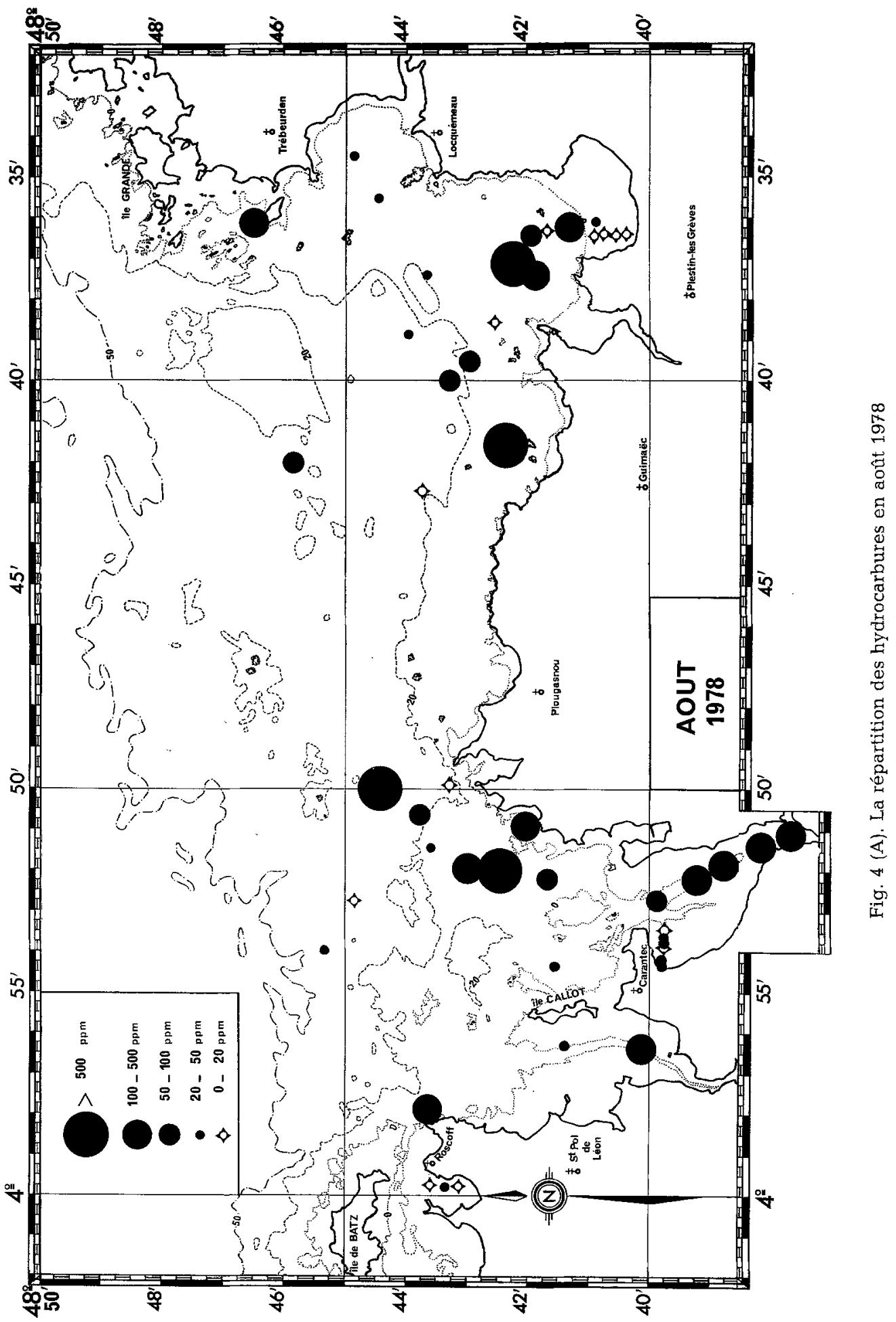




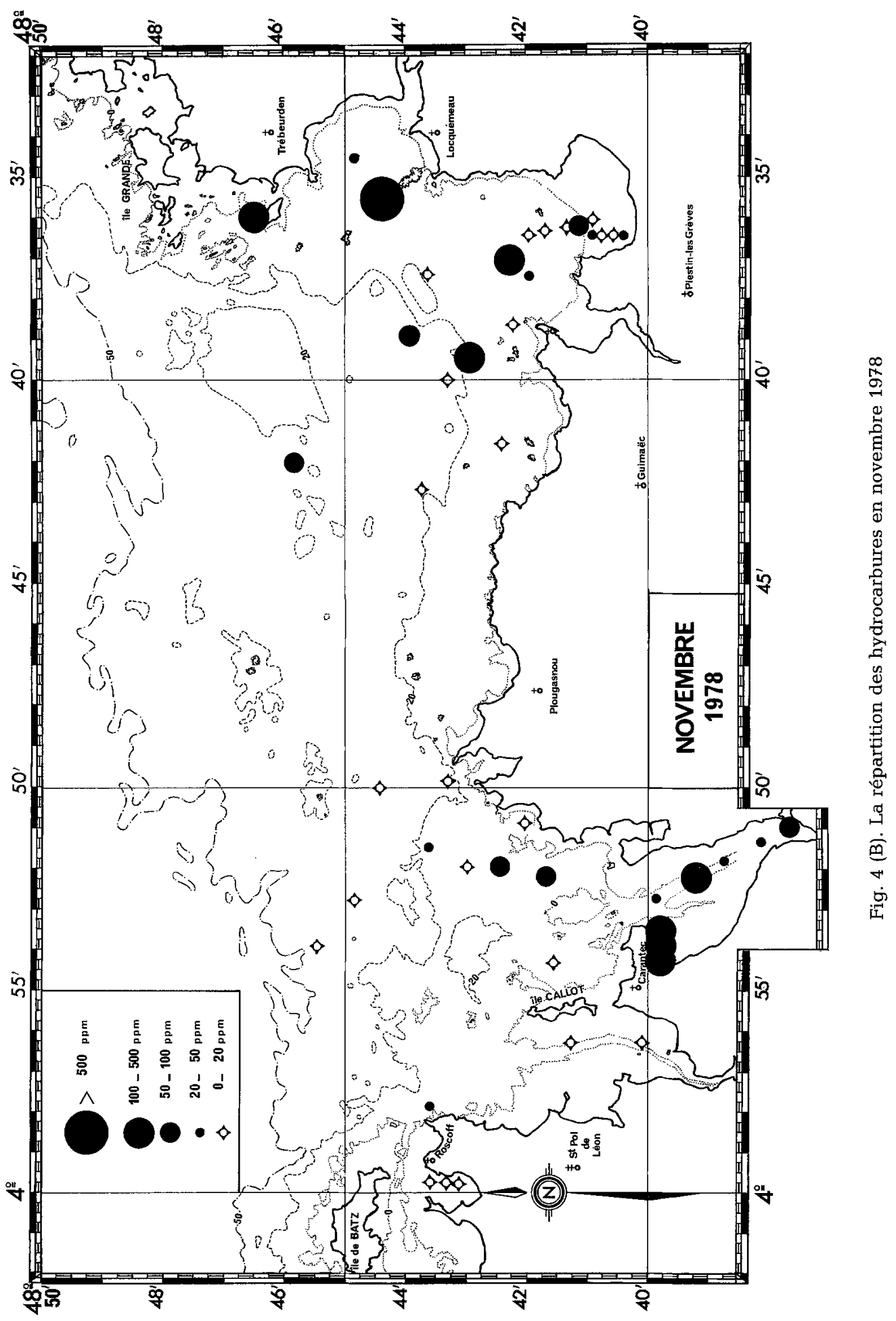


La pollution des Baies de Morlaix et de Lannion

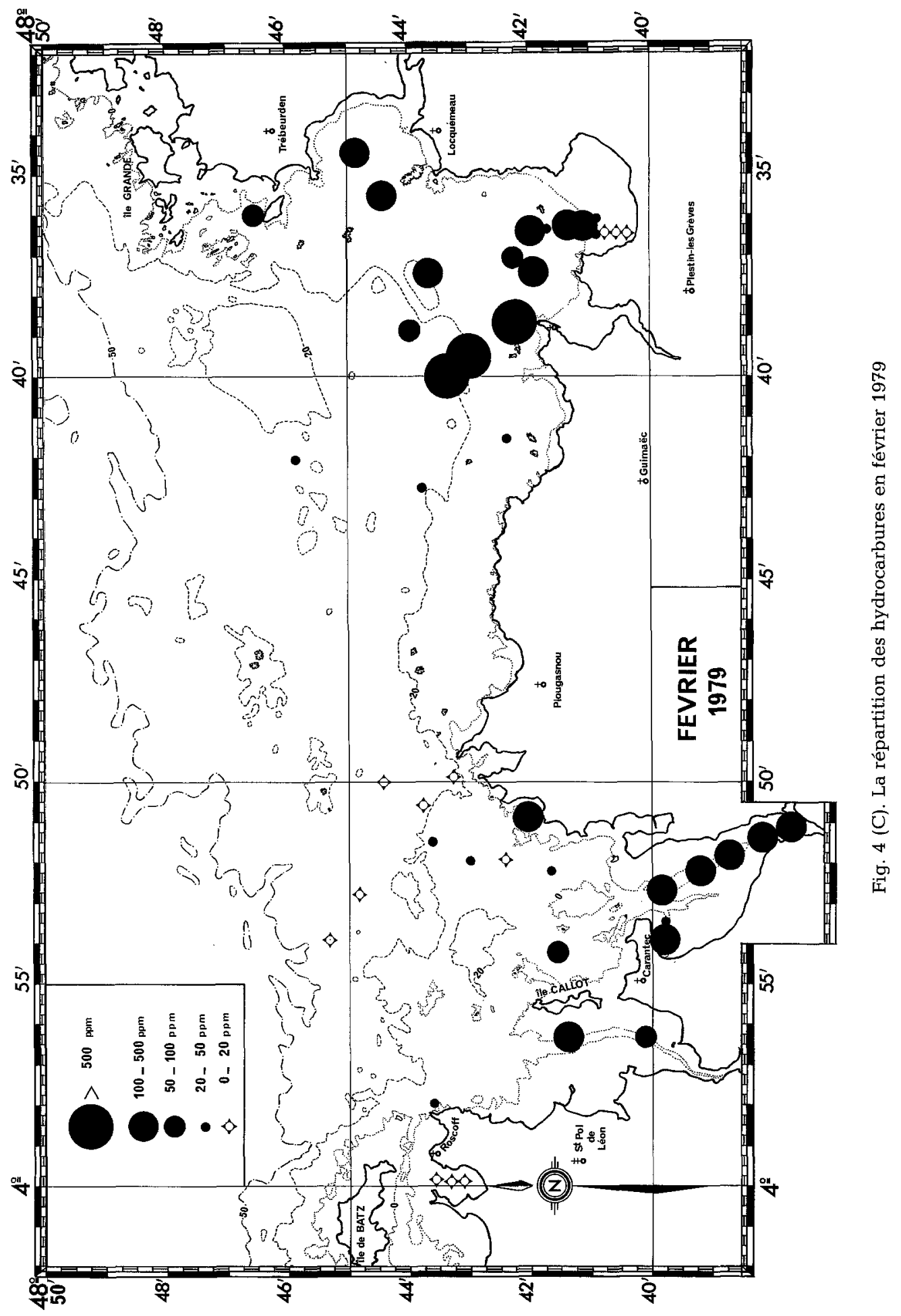




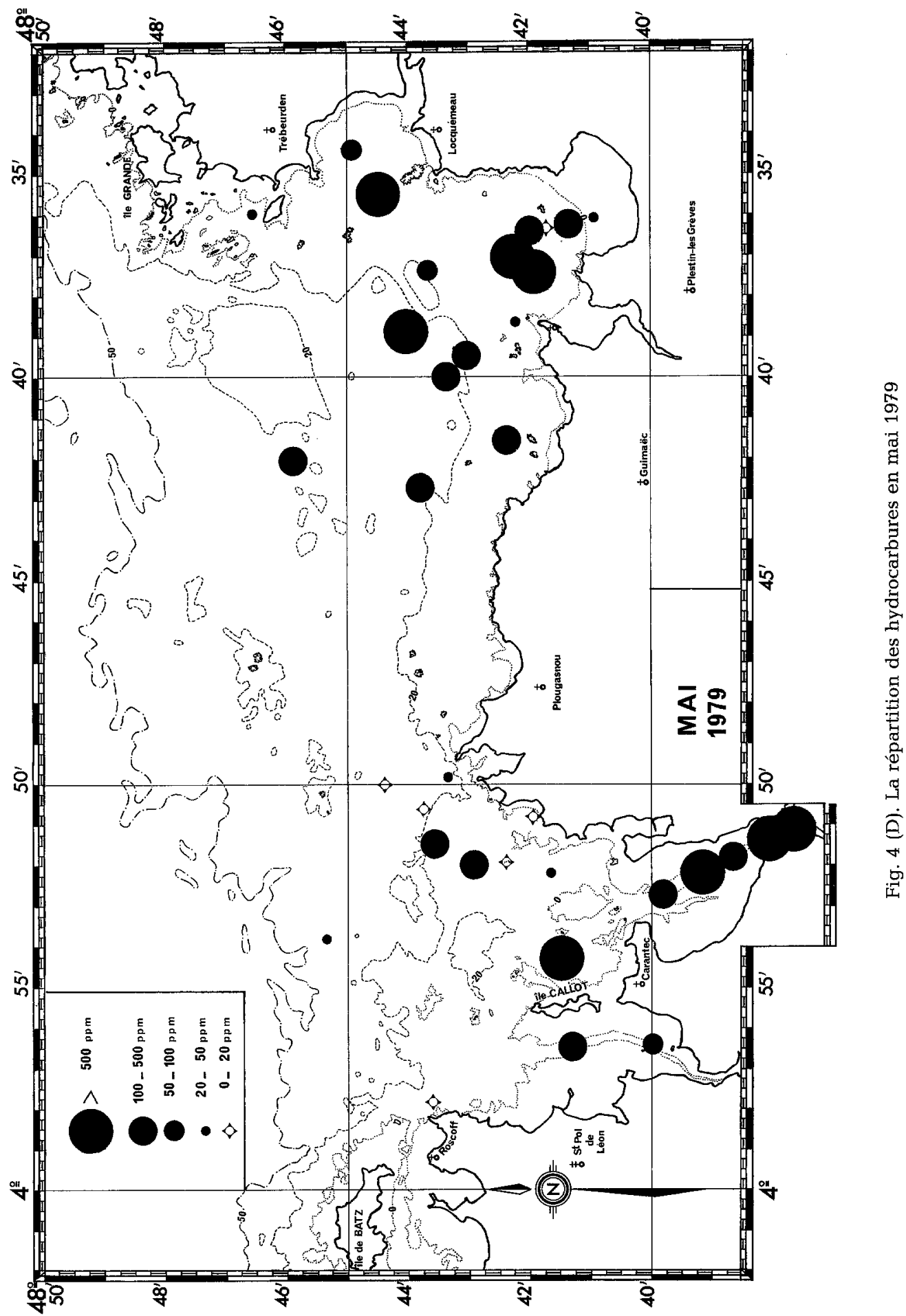


mer n'a été fort que 2 fois 2 jours en septembre-octobre (Fig. 5). Dans ces conditions, l'action mécanique des houles, très efficace sur la dispersion du mazout et par conséquent sur la dépollution (Owens, 1978) ne s'est exercée que modérément, à proximité du rivage. Dans les zones du large, la décontamination constatée doit être principalement attribuée à l'agitation provoquée par les courants de marée et aux processus d'oxydation et de biodégradation s'exerçant progressivement sur les hydrocarbures incorporés au sédiment. Dans l'ensemble, les faibles remaniements provoqués par les courants de marée ou par les houles ont alimenté les suspensions qui sont allées se décanter dans les zones les plus calmes (estuaires) ou pénétrer dans les interstices des fonds à Lithothamniées (maerl envasé fortement contaminé de la région de Locquémeau-Trébeurden par exemple). Il apparaît donc que les zones de basse énergie où la décontamination par action mécanique est très faible constituent des sites privilégiés pour la fixation et le stockage des hydrocarbures résiduels. Ce phénomène qui conduit à l'accroissement des teneurs relevées est encore amplifié par le fait que la biodégradation n'intervient que faiblement dans ces dépôts fins peu oxygénés.
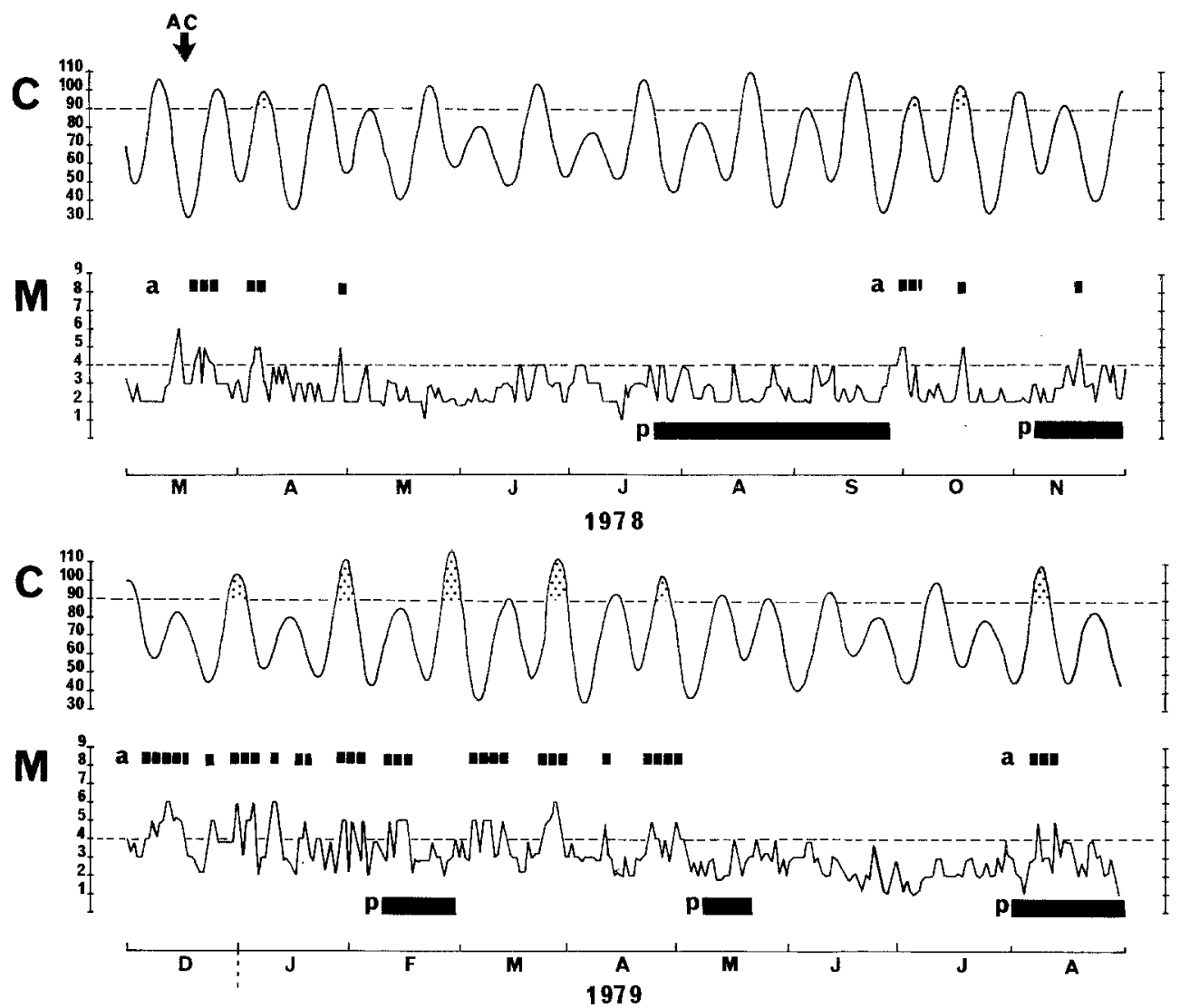

Fig. 5. Cycle des marées et états de la mer entre l'échouage de l' Amoco Cadiz" et le mois d'août 1979. C: Coefficient de marée, M: Etat de la mer (échelle de Beaufort), AC: Echouage de l'"Amoco Cadiz", P: Période de prélèvements, a: Période de forte agitation, petits points: Période de coïncidence vive eau et mauvais temps 
Les résultats de février 1979 (Fig. 4c) indiquent une nette évolution par rapport aux précédents. Si les hydrocarbures ont disparu des zones de forte énergie, les régions calmes à très calmes présentent une forte pollution, beaucoup plus élevée qu'en novembre 1978. Ce résultat apparaît nettement dans la rivière de Morlaix et plus encore dans l'anse du Guerzit et dans la baie de Lannion où la pollution de l'ordre de 250 ppm atteint les sablons jusqu'à une vingtaine de mètres de profondeur. La campagne de mai 1979 (Fig. 4d) montre une accentuation des faits observés en février. On relève alors de très fortes concentrations dans la rivière de Morlaix (jusqu'à $3000 \mathrm{ppm}$ ) et dans la baie de Lannion (200 à $1000 \mathrm{ppm}$ ). La pollution s'étale un peu plus vers le large, affecte à nouveau les sédiments fins de la région de la Pierre Noire et s'accumule toujours dans le maerl envasé (abords de l'île Callot et $\mathrm{N}$ de Locquémeau). A l'exception de ces dépôts particuliers de maerl envasé on constate une étroite corrélation entre les teneurs en hydrocarbures et l'importance de la fraction fine des sédiments. Les données météorologiques permettent d'interpréter les résultats (Fig. 5).

A partir de décembre 1978, de longues périodes de mauvais temps ont été relevées, coïncidant parfois avec de fortes marées (fin décembre par exemple). La turbulence élevée des eaux a engendré d'importants remaniements en domaine subtidal peu profond et surtout sur la frange côtière. Les déchets de mazout abandonnés par les nappes de l' "Amoco Cadiz" au printemps 1978 et non récupérés ont été partiellement remobilisés entraînant le nettoyage superficiel des plages exposées aux assauts des vagues. Les réservoirs massifs de pollution que constituent les abers ont également pu libérer vers l'extérieur une part de leur charge en mazout. Après un transport en suspension plus ou moins long s'effectuant d'Ouest en Est en fonction des dérives résiduelles, le matériel oléosédimentaire, plus ou moins dégradé, trié, est allé s'accumuler dans les zones de faible énergie ou se concentrer dans les pièges biologiques comme le maerl envasé. Ainsi, un an après la catastrophe en baies de Morlaix et de Lannion, certaines zones subtidales sont-elles plus polluées, qu'au printemps 1978. Tant que des réservoirs de pollution subsisteront, ils seront susceptibles d'alimenter les suspensions et de contaminer les fonds de vases et de sablons.

\section{CONCLUSION}

L'étude de la distribution des hydrocarbures de 1"'Amoco Cadiz' dans les sédiments et de son évolution, au cours de l'année faisant suite à la catastrophe, conduit à proposer le schéma interprétatif suivant.

En premier lieu, le déversement massif de pétrole coïncidant avec les tempêtes de la fin de la mauvaise saison a entraîné la pollution brutale de l'environnement marin à la fois en domaine intertidal et en milieu subtidal.

Dans la zone de balancement des marées, les produits mazoutés se sont répartis par échouage en relation avec le régime des vents. Plus au large, la contamination de fonds de nature variée par du matériel oléo-sédimentaire résulte du délestage d'épaisses nappes de pétrole suite à l'accumulation en surface et à leur émulsification partielle en milieu turbulent.

Parallèlement, le transport en suspension des particules polluées sous l'action des agents hydrodynamiques locaux , les courants de marée principalement, a provoqué la contamination des sédiments fins de la région. 
Par la suite, les hydrocarbures imprégnant les sédiments ont subi des phénomènes de dégradation et de remaniement. Pendant la longue période de calmes estivaux, jusqu'en novembre 1978, la tendance générale a été à la décontamination sous l'action de la biodégradation et de la dispersion par les courants de marée, les fonds soumis à l'agitation la plus forte évoluant le plus rapidement. Seules des zones calmes d'accumulation ont révélé une augmentation des teneurs.

Pendant la période des tempêtes hivernales et printanières, jusqu'en mai 1979, les fortes houles ont provoqué le brassage des sédiments côtiers, nettoyé la majeure partie du littoral et libéré de nouvelles charges d'hydrocarbures plus ou moins dégradés. Remis en suspension, ce matériel a été dispersé par les courants pour aller s'accumuler dans les zones de basse énergie occupées par des sablons et des sédiments vaseux ou s'incorporer à certains dépôts hétérogènes envasés. Il est probable que le matériel ainsi accumulé ne représente pas la totalité des suspensions libérées par les remaniements côtiers, une partie étant sans doute entraînée vers le large et suffisamment dispersée par les dérives générales de la Manche pour n'être plus détectable. Les phénomènes que nous avons suivis au cours de la première année sont susceptibles, compte-tenu des cycles météorologiques saisonniers, de se reproduire à l'avenir en s'atténuant progressivement.

Dans le cas présent, leur déroulement a été conditionné par la structure océanographique de la région marquée par une large prédominance des zones de haute énergie dynamique (houles, courants de marée), très favorable à la dispersion et à la dégradation des hydrocarbures. La pollution à moyen et long terme n'affecte que des étendues relativement limitées correspondant aux zones calmes d'accumulation. Il est probable qu'une pollution du même ordre se produisant dans un milieu dans l'ensemble moins turbulent aurait une évolution plus lente.

Remerciements. Les travaux ont été effectués dans le cadre du Groupe de Recherches coordonnées "Manche" (G. R. E. C. O. du Centre National de la Recherche Scientifique). Ils s'intègrent au programme national de recherches entrepris après l'échouage de l' "Amoco Cadiz" et coordonné par le Centre National pour l'Exploitation des Océans (opérations financées d'une part par le Ministère de l'Environnement et du Cadre de Vie, d'autre part par la National Oceanographic \& Atmospheric Administration des U. S. A.). Les auteurs remercient le Service Hydrographique et Océanographique de la Marine pour l'aide qu'il a apportée dans l'exécution du programme, par le détachement de M. Marc Richard à la Station Biologique de Roscoff au titre de son service national. Ils témoignent également leur reconnaissance à la Mutuelle Générale de l'Education Nationale qui leur a financé une partie des équipements de mesure. Ils remercient enfin les équipages des navires de la Station Biologique de Roscoff pour leur amicale et efficace collaboration.

\section{LITTÉRATURE CITÉE}

Allen, G., D'Ozouville, L. \& l'Yavanc, J., 1978. Etat de la pollution par les hydrocarbures dans l'Aber Benoît. - Publs CNEXO (Actes de Colloques) 6, 97-114.

Anonymus, 1968. Courants de marée dans la Manche et sur les côtes françaises de l'Atlantique. Serv. hydrogr. mar, 550, 1-276.

Auffret, G. \& Douvillé, J. L. de, 1974. Observations sur la dynamique des sables de la Pierre Noire (baie de Morlaix, Finistère). - Bull. Bur. Rech. géol. Minières (Sér. 2, sect. 4) 1, 5-18.

Beynon, R., Kashnitz, R. \& Rijnders, G., 1968. Methods for the analysis of oil in water and soil. Stichting Concave, The Hague, 1-51.

Berné, S., Brossier, R., Fontanel, A., d'Ououville, L., Serriere, J. \& Wadsworth, A., 1978. Télédétection des pollutions par hydrocarbures de l'"Amoco Cadiz". - Publs CNEXO, (Actes de Colloques) $6,9-26$. 
Boillot, G., 1964. Géologie de la Manche occidentale. - Annls Inst. océanogr. Monaco 42, 1-220.

Boucher, G., 1979. Impact of the "Amoco Cadiz" oil spill on intertidal and sublittoral sands. - Mar. Pollut. Bull. (In press.)

Cabioch, L., 1968. Contribution à la connaissance des peuplements benthiques de la Manche occidentale. - Cah. Biol. mar. 9, 493-720.

Cabioch, L., Dauvin, J. C. \& Gentil, F., 1978. Preliminary observations on pollution of the sea-bed and disturbance of sub-littoral communities in Northern Brittany by oil from the "Amoco Cadiz". - Mar. Pollut. Bull. 9, 303-307.

Cabioch, L. \& Douvillé, J. L., 1979. La circulation des eaux dans la baie de Morlaix et ses abords: premières données obtenues par suivis de flotteurs dérivants. - Trav. Stn biol. Roscoff (N. S.). (Sous presse.)

Causse, C. \& Mestres, R., 1975. Dosage des résidus d'hydrocarbures par infra-rouge dans l'eau de mer et les sédiments marins. In: Manuel des méthodes de prélèvement et d'analyse. Réseau national d'observation de la qualité du milieu marin. CNEXO, Brest, 2, 13-18.

Douvillé, J. L., 1975. Cartographie des intensités maximales des courants de marée au large de Roscoff. - Trav. Stn biol. Roscoff (N. S.) 22, 21-26.

D'Ozouville, L., Gundlach, E. R. \& Hayes, M. O., 1978. Effect of coastal processes on the distribution and persistence of oil spilled by the "Amoco Cadiz". Preliminary conclusions. - Publs CNEXO, (Actes de Colloques) 6, 69-96.

Gundlach, E. R. \& Hayes, M. O., 1978. The "Amoco Cadiz" oil spill.-Spec. Rep. Research Planning Institute, Columbia, South Carolina 3, 1-67.

Gundlach, E. R. \& Hayes, M. O., 1979. The "Amoco Cadiz" oil spill. - Spec, Rep. Research Planning Institute, Columbia South Carolina 4, 1-30.

Hayes, M. O. \& Gundlach, E. R., 1979. Beach processes and oil sediment interaction of the "Amoco Cadiz" oil spill site. 145th natn Meet. Am. Ass. Adv. Sci. Abstr. 43.

Hiltabrand, R. R., 1978. Estimation of aromatic hydrocarbon in sea-water at proposed Deep Water Port (DWP) sites in the Gulf of Mexico. - Mar. Pollut. Bull. 9, 19-21.

Huang, C. \& Elliott, H. A., 1977. The stability of emulsified crude oil as affected by suspended particles. In: Fate and effects of petroleum hydrocarbons in marine organisms and ecosystems. Ed. by D. A. Wolfe. Pergamon Press, Oxford, 413-420.

Jones, S. R., 1974. Some results from woodhead sea-bed drifter releases in the western English Channel and Celtic Sea. - Tech. Rep. Fish. Lab., Lowestoft 10, 1-21.

Larsonneur, C., 1972. Le modèle sédimentaire de la Baie de Seine à la Manche Centrale dans son cadre géographique et historique. Colloque sur la géologie de la Manche. - Mém. Bur. Rech. géol. Minière $79,241-255$.

Larsonneur, C., 1977. La cartographie des dépôts meubles sur le plateau continental français: méthode mise au point et utilisée en Manche. - J, Rech. océanogr. 2, 33-39.

Larsonneur, C., Vaslet, D. \& Auffret, J. P., 1978. La carte des sédiments superficiels de la Manche au 1/500 000. Bur. Rech. géol. Minières, Orléans.

MacAuliffe, C. D., 1977. Dispersal and alteration of oil discharged on a water surface. In: Fate and effects of petroleum hydrocarbons in marine organisms and ecosystems. Ed. by D. A. Wolfe. Pergamon Press, Oxford, 19-35.

Marchand, M. \& Caprais, M. P., 1979. Suivi chimique de la pollution de "l'Amoco Cadiz" dans l'eau de mer et les sédiments en Manche occidentale. - Rapp. CNEXO-COB, 1979, 1-101.

Owens, E. H., 1978. Mechanical dispersal of oil stranded in the littoral zone. - J. Fish. Res. Bd Can. $35,563-572$. 